\title{
LPV model-based robust diagnosis of flight actuator faults ${ }^{\underline{t h}}$
}

\author{
Andreas Varga*, Daniel Ossmann \\ German Aerospace Center, DLR-Oberpfaffenhofen, Institute of System Dynamics and Control, D-82234 Wessling, Germany
}

\begin{abstract}
A linear parameter-varying (LPV) model-based synthesis, tuning and assessment methodology is developed and applied for the design of a robust fault detection and diagnosis (FDD) system for several types of flight actuator faults such as jamming, runaway, oscillatory failure, or loss of efficiency. The robust fault detection is achieved by using a synthesis approach based on an accurate approximation of the nonlinear actuator-control surface dynamics via an LPV model and an optimal tuning of the free parameters of the FDD system using multi-objective optimization techniques. Real-time signal processing is employed for identification of different fault types. The assessment of the FDD system robustness has been performed using both standard Monte Carlo methods as well as advanced worst-case search based optimization-driven robustness analysis. A supplementary industrial validation performed on the AIRBUS actuator test bench for the monitoring of jamming, confirmed the satisfactory performance of the FDD system in a true industrial setting.
\end{abstract}

Keywords: Fault detection, fault identification, fault diagnosis, flight actuator faults.

\section{Introduction}

There are several classes of flight actuator faults, such as jamming, runaway, oscillatory failure or loss of efficiency, whose early detection and timely handling contribute to the efficient operation of aircraft, avoid excessive fuel consumption (with all associated negative environmental effects) and increase the aircraft operational autonomy. Therefore, the deployment of fault detection and diagnosis (FDD) systems for monitoring and identification of these type of faults is of paramount importance for large civil transport aircraft. Once a certain type of fault has been identified, appropriate reconfiguration actions may take place in order to eliminate the effects of malfunctioning and ensure acceptable performance. To fulfill standard certification requirements, the flight control system augmented with appropriate FDD systems must reliably operate over the whole flight envelope, for a large variety of maneuvers, in the presence of uncertainties as external wind disturbances or variations of aircraft parameters.

The synthesis methodologies of FDD systems for various flight actuator failures must comply with these strong requirements, by providing designs which guarantee performance robustness (e.g., lack of false alarms, lack of missed detections, fast detection times) in all operating conditions. In the current industrial practice, threshold based signal monitoring schemes are widely used in the aircraft industry (Goupil, 2011). The use of first order linear time-invariant (LTI) actuator models for

\footnotetext{
This work was performed in the framework of the European ADDSAFE Project: Grant agreement no.: FP7-233815.

${ }^{*}$ Corresponding author.

Email addresses: andreas.varga@dlr.de (Andreas Varga), daniel.ossmann@dlr.de (Daniel Ossmann)
}

model-based fault detection purposes has been also considered. However, because LTI models completely ignore the complex dependency between the actuator dynamics and the aerodynamics forces acting on the control surfaces, their use may raise difficulties in guaranteeing the lack of false alarms over a wide range of variation of flight parameters and in the presence of large parameter variations. Recently proposed advanced techniques based on the use of the so-called linear parameter varying (LPV) models are therefore potentially better suited for guaranteeing robustness of fault detection performance, as illustrated in several recent works (Varga et al., 2011b; Alwi and Edwards, 2012; Henry et al., 2012; Vanek et al., 2012; Varga and Ossmann, 2012). Fault identification aspects have been also recently considered in several works, as for example the oscillatory failure case in (Goupil, 2010; Alcorta-Garcia et al., 2011; Varga and Ossmann, 2012) as well as the actuator jamming and runaway in (Varga et al., 2011b; Gheorghe et al., 2013).

In this paper an LPV model-based synthesis, tuning and assessment methodology is described for the development of robust FDD systems for detection and identification of flight actuator faults. This methodology is illustrated in four actuator/control surface failure cases: jamming, oscillatory failure, runaway and loss of efficiency. The robustness of fault detection is achieved by using a synthesis method based on an accurate approximation of the nonlinear actuator-control surface dynamics via a LPV model in conjunction with an optimal tuning of the free parameters (e.g., thresholds) of the FDD system components using multi-objective optimization techniques. Signal processing algorithms are employed for on-line identification of specific faults. The assessment of the FDD system robustness has been performed using both standard Monte Carlo methods as well as advanced worst-case search based 
optimization-driven robustness analysis. A supplementary industrial validation of the FDD system for monitoring of jamming has been performed on the AIRBUS actuator test bench in the framework of the ADDSAFE project and confirmed the satisfactory performance of the FDD system in a true industrial setting (Varga et al., 2013).

The structure of the paper is explained in what follows. In Section 2, a generic FDD system architecture is proposed for the solution of the fault detection, isolation and identification problems for the most important classes of actuator faults mentioned above. A model-based synthesis methodology of the components of the FDD system is described in Section 3, addressing the development of a suitable LPV-model for the actuator dynamics (Section 3.1), the synthesis of the residual generator (Section 3.2), the setup and tuning of residual evaluation and decision making blocks (Section 3.3), the choice of the fault identification algorithms (Section 3.4), and the assessment of the FDD system robustness (Section 3.5). The application of this methodology for the design of a robust FDD system for monitoring four types of actuator faults is described in Section 4.

\section{FDD system architecture for monitoring flight actuator faults}

For the model-based monitoring of flight actuator faults two approaches are in principle possible. The system-wide approach employs a unique FDD system which relies on a fault detection filter (or residual generator) which processes the set of known signals (output measurements, control inputs, scheduling parameter values) and produces a structured residual vector, whose zero-nonzero components provide information on the presence or absence of actuator faults (fault detection) as well as on their localization (fault isolation). The fault detection and isolation (FDI) is usually followed by fault identification, to determine the nature of the occurred fault and its essential characteristics, which can serve for control law reconfiguration purposes. This approach has the potential advantage to handle all actuator faults (possibly jointly with sensor faults) using a single fault detection filter. Also, all categories of actuator faults can be detected, independently of the availability of additional control surface sensors. However, the complexity of the underlying nonlinear aircraft model and the presence of various uncertainties (e.g., unknown wind disturbances, aircraft parameter variations, uncertain aerodynamical coefficients) make the synthesis of a reliable FDD system very challenging (if not impossible). The feasibility of this approach has been investigated in (Varga, 2009b), where only the nominal case has been considered. Even in this case, the presence of multiple control surfaces as is typical for elevators, rudders or ailerons, make the fault isolation task very challenging in presence of model uncertainties.

In a component level FDD approach, each actuator is independently monitored, using exclusively locally available signal measurements, as the position of the actuator rod, the surface angle deflection, and the generated control input representing the commanded surface position. The main advantage of such local approach is that fault isolation is implicitly provided by fault detection. Simple to implement (first or second order) fault detection filters can be employed to detect several types of additive faults, as jamming, oscillatory failure, runaway and a class of loss of efficiency failure. Robustness aspects can be addressed by employing, for example, gain scheduling techniques based on LPV-models. Fault identification can be also easily performed applying signal processing techniques on the available signals (also including the residual signals). The component level approach has difficulties in detecting other fault types (e.g., multiplicative), as for example, the loss of effectiveness due to surface disconnection (e.g., broken actuator rod) or control surface damage. In these cases, a combined fault detection and identification approach based on model detection techniques (see Varga, 2009a; Ossmann and Varga, 2013) can be employed to estimate the level of the loss of efficiency, by relying on suitable multiple fault models describing the actuatorcontrol surface aerodynamic interactions.

The block-diagram of the component level FDD approach is depicted in Fig. 1, where we assume that the FDD system works jointly with a stable closed-loop flight control system, whose main components are the open-loop aircraft, the controller, the actuator and sensor blocks. The controller processes the pilot command vector $\underline{u}_{p}(t)$ used to perform various maneuvers and the measured outputs vector $\underline{y}_{m}(t)$ delivered by the sensors using the aircraft output vector $y(t)$, and produces the actuator command vector $\underline{u}_{c}(t)$. The actuator output signals form the vector $\underline{u}(t)$ representing the corresponding surface deflections. The FDD system, whose detailed structure is presented in Fig. 2, processes a single actuator output signal $u(t)$ and the corresponding actuator command $u_{c}(t)$, which are selected via the selector block from the components of the actuator output vector $\underline{u}(t)$ and actuator command vector $\underline{u}_{c}(t)$, respectively. Additionally, the aircraft parameters $p(t)$ are used for gain scheduling purposes. The output of the FDD system is a vector $\eta(t)$ of confirmation signals, which indicate the presence or absence of specific actuator faults.

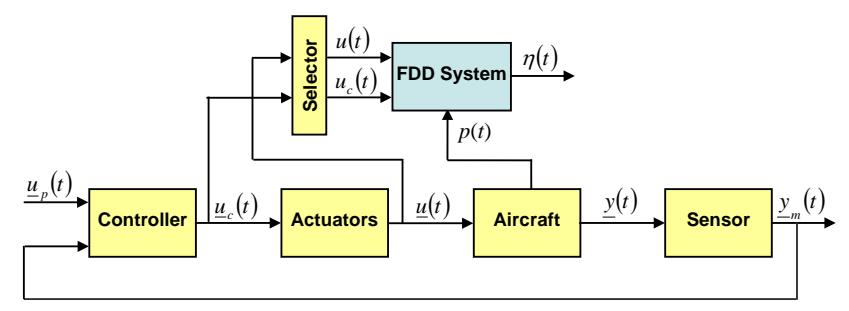

Figure 1: Fault monitoring setup for FDD of a single actuator

The FDD system used in this paper to detect and identify different types of actuator faults is depicted in Fig. 2 and is based on the local approach already described in (Varga et al., 2011b). The FDD system structure in Fig. 2 only includes a single residual generator for fault detection (or simply, a fault detector), which processes the commanded actuator position $u_{c}$ and the measured current actuator position $u$ and generates the scalar residual signal $r$. For the robust fault detection, a single LPV 
detector is sufficient, where $\rho$ is a vector whose components are the scheduling variables as described in Section 3.1.

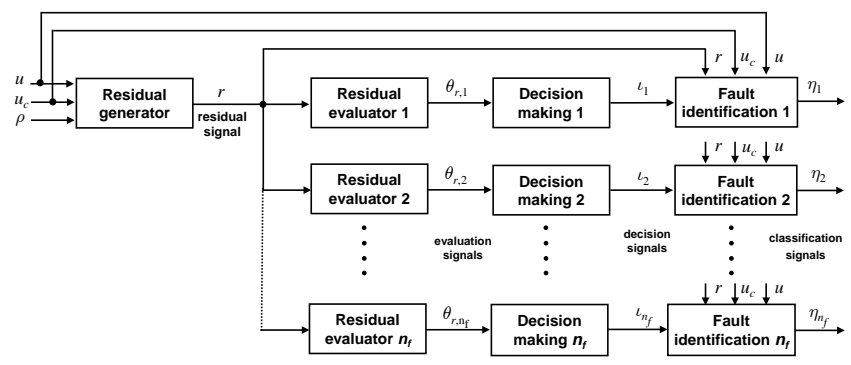

Figure 2: FDD system architecture for actuator fault identification

For the identification of different types of faults, individual post-processing of the residual is performed, by taking into account the different possible shapes of fault signals as well as the specific requirements on detection times. For $n_{f}$ different types of fault signals to be detected, a multi-channel structure is used for the residual evaluation, decision making and fault identification. For each fault type to be identified, a residual evaluation block and decision making block are present, whose parameters are chosen to reflect the characteristics of the assumed fault signal. Thus, for the $j$-th type of fault, a specific approximation $\theta_{r, j}$ of the residual norm $\|r\|_{2}$ is computed in the corresponding $j$-th residual evaluator block. Then, this value is used in the threshold-based decision making block to generate the decision signal $\iota_{j}$, which, if nonzero, triggers a signal based fault identification process. Each identification block provides qualitative and quantitative information on the faults by processing the input and output signals $u_{c}$ and $u$ of the actuator, as well as the residual signal $r$. The output of this block is the fault classification signal $\eta_{j}$, which can be used to trigger specific control reconfiguration actions.

\section{Model-based synthesis methodology}

In this section we describe a model-based synthesis methodology of FDD systems having the generic architecture depicted in Fig. 2 for the detection, isolation and identification of flight actuator faults. This methodology involves several main steps, which are described subsequently:

1. Development of a suitable LPV synthesis model of the underlying actuator;

2. Synthesis of a LPV residual generator for robust fault detection;

3. Setting up of the residual evaluation and decision making blocks;

4. Development of signal processing based fault identification schemes;

5. Assessment of the robustness of the FDD system.

This methodology is primarily intended for the robust fault monitoring of hydraulic servo controlled actuators which are widely used in today's aircraft Goupil (2010). The key aspects in this context are the use of LPV-residual generators based on accurate LPV-models of the actuator, the optimization-based tuning of the parameters of the residual evaluation blocks, and the use of efficient signal processing based fault identification techniques.

\subsection{LPV model generation for the nonlinear actuator model}

The development of an accurate approximation of the joint actuator and control surface dynamics in the form of a quasiLPV-model is an important prerequisite for the synthesis of a robust FDD system for monitoring actuator faults. For the details on the derivation of such quasi-LPV models see (Varga et al., 2011b). The resulting actuator model has a first order LPV-system representation of the form

$$
\dot{u}=-k(\rho) u+k(\rho) u_{c},
$$

where $u$ is the rod position (or equivalently the surface deflection angle) and $u_{c}$ is the commanded position (deflection angle). The gain $k(\rho)$ generally depends on several measurable parameters grouped into a vector $\rho$.

The underlying actuator model is a simplified nonlinear dynamic model of an hydraulic servo controlled actuator described by a first order nonlinear state equation of the form (Goupil, 2010; Márton and Ossmann, 2012)

$$
\dot{u}=K(p, u, \dot{u})\left(u_{c}-u\right)
$$

with the nonlinear gain

$$
K(p, u, \dot{u}):=K_{c i} K_{p} \sqrt{\frac{\Delta P(u)-\frac{F_{a e r o}(p, u, \dot{u})+K_{d} \dot{u}^{2}}{S}}{\Delta P_{r e f}}},
$$

where $K_{p}$ is the servo control gain, $K_{c i}$ is a gain to convert an estimated current to a corresponding rod speed, $\Delta P$ is the hydraulic pressure delivered to the actuator, $\Delta P_{\text {ref }}$ is a differential pressure for a fully opened servo-valve (maximum rod speed), $F_{\text {aero }}$ represents the aerodynamic forces at the control surface, $S$ is the actuator piston surface area and $K_{d} \dot{u}^{2}$ represents the estimated servo-control load of the adjacent actuator in damping mode (only in the case of two actuators per control surface in a dual active/passive scheme). The components of the vector $p$ are the relevant aircraft parameters and flight conditions.

To get a simple quasi-LPV model approximating (2) with good accuracy, the nonlinear gain (3) is approximated by an easily computable gain $k(p, u, \dot{u})$, which is then used in the first order actuator model as given in (1). The main variations of $K$ are caused by the aerodynamic force $F_{\text {aero }}$ that acts on the control surface, where $F_{\text {aero }}$ itself usually depends on the parameters in $p$, the actuator position $u$ and the sign of the actuator position rate $\dot{u}$. The effect of these variations is a reduction or increase of the gain, and thus variations of the response speed of the actuator. More details on the physical interpretation of $F_{\text {aero }}$ can be found in (Varga et al., 2011b).

Physical considerations as described in (Varga et al., 2011b) led to choosing $k(p, u, \dot{u})$ of the form

$$
k(p, u, \dot{u})=C_{0}(p)+C_{1}(p) \operatorname{sign}(\dot{u})\left(u+C_{2}(p)\right),
$$


where for fixed $p, C_{0}(p)$ can be interpreted as the nominal gain, $C_{1}(p)$ describes the influence of the deflection angle $u$ on $k$, while the factor $\operatorname{sign}(\dot{u})$ allows to distinguish between upward and downward movements of the control surface. $C_{2}(p)$ can be interpreted as a position offset. The chosen functional dependence on $u$ and $\operatorname{sign}(\dot{u})$ reflects the actual physical behavior of the actuator for different control surface positions and signs of deflection rate. For $C_{i}(p), i=0,1,2$, affine approximations have been used, where the intervening constant coefficients have been determined using parameter fitting techniques based on comparing the output responses of the nonlinear actuator model (2) and LPV-model (1). The final form of $k(\rho)$, with $\rho=(u, \operatorname{sign}(\dot{u}), p)$ is simple enough to be used in LPVmodel based fault diagnosis applications. The accuracy of this model can be improved for large surface deflections, by taking into account saturations occurring at its blowdown limit, which is reached when the sum of the force from the air (or blowdown) $F_{\text {aero }}$ and the damping force $K_{d} \dot{u}^{2}$ equals the maximum available force due hydraulic pressure $\Delta P \cdot S$.

Alternative quasi-LPV actuator models have been derived in (Henry et al., 2012) and (Vanek et al., 2012), where instead of approximating $K(p, u, \dot{u})$ directly, first an approximation of $F_{\text {aero }}(p, u, \dot{u})$ has been constructed. The generation of the final LPV models involves further rational/polynomial approximations in order to arrive to a form suitable for the applicability of employed synthesis methodologies.

\subsection{Synthesis of an $L P V$ residual generator}

Assume temporarily that the parameters in $\rho$ are constant. In this case an input-output representation of the actuator fault model is used in the additive form

$$
\mathbf{u}(s)=G(s, \rho) \mathbf{u}_{c}(s)+G_{f}(s, \rho) \mathbf{f}(s),
$$

where $\mathbf{u}(s), \mathbf{u}_{c}(s)$, and $\mathbf{f}(s)$ are the Laplace-transformed quantities of $u(t), u_{c}(t)$, and additive fault signal $f(t)$, respectively, and $G(s, \rho)$ and $G_{f}(s, \rho)$ are parameter dependent transfer functions. $G(s, \rho)$ corresponding to (1) is

$$
G(s, \rho)=\frac{k(\rho)}{s+k(\rho)},
$$

while $G_{f}(s, \rho)=G(s, \rho)$ for an input located fault (assumed in this paper).

As residual generator a parameter dependent filter is used of the form

$$
\mathbf{r}(s)=Q(s, \rho)\left[\begin{array}{c}
\mathbf{u}(s) \\
\mathbf{u}_{c}(s)
\end{array}\right],
$$

where $Q(s, \rho)$ is the $1 \times 2$ transfer-function matrix of the filter, which explicitly depends on the measurable parameter $\rho$ (e.g., via an equivalent state-space realization of the filter). For a physically realizable filter, $Q(s, \rho)$ must be robustly stable with respect to $\rho$. The robust fault detection synthesis problem addresses the robustness of the fault detection system with respect to the measurable parameter $\rho$ by attempting to achieve robustness using an LPV gain scheduling approach.
To address the robust fault detection problem, the synthesis method described in (Varga, 2011; Varga et al., 2011b) has been employed. A first order detector is used having the form

$$
Q(s, \rho)=\left[\begin{array}{ll}
\frac{a}{k_{0}} \frac{s+k(\rho)}{s+a} & -\frac{k(\rho) a}{k_{0}(s+a)}
\end{array}\right],
$$

where $a$ is an arbitrary positive value specifying the dynamics of the detector and $k_{0}$ is a typical nominal value of the gain $k(\rho)$. By replacing in (7) $\mathbf{u}(s)$ by its expression in (5), the internal form of the detector is obtained

$$
\mathbf{r}(s)=R(s, \rho) \mathbf{u}_{c}(s)+R_{f}(s, \rho) \mathbf{f}(s),
$$

where

$$
\left[R(s, \rho) \mid R_{f}(s, \rho)\right]:=Q(s, \rho)\left[\begin{array}{c|c}
G(s, \rho) & G_{f}(s, \rho) \\
1 & 0
\end{array}\right]
$$

The choice (8) of $Q(s, \rho)$ guarantees an exact decoupling of control inputs in (10), thus $R(s, \rho)=0$. The corresponding fault-to-residual transfer function is

$$
R_{f}(s, \rho)=\frac{k(\rho)}{k_{0}} \frac{a}{s+a}
$$

Thus, the residual signal provides a filtered estimation of the fault, allowing to easily reconstruct the actuator fault signal $f$ for further use in fault identification.

The LPV state-space realization of the residual generator (7) can be always obtained in the form

$$
\begin{aligned}
\dot{x}_{Q}(t) & =A_{Q} x_{Q}(t)+B_{Q}(\rho)\left[\begin{array}{c}
u(t) \\
u_{c}(t)
\end{array}\right] \\
r(t) & =C_{Q} x_{Q}(t)+D_{Q}(\rho)\left[\begin{array}{c}
u(t) \\
u_{c}(t)
\end{array}\right]
\end{aligned}
$$

For the detector (8), the state-space matrices are

$$
\begin{aligned}
& A_{Q}=-a, \quad B_{Q}(\rho)=a\left[\frac{k(\rho)-a}{k_{0}}-\frac{k(\rho)}{k_{0}}\right], \\
& C_{Q}=1, \quad D_{Q}=\left[\begin{array}{cc}
\frac{a}{k_{0}} & 0
\end{array}\right] .
\end{aligned}
$$

The chosen form (8) of the detection filter leads to a state-space realization (11) with a constant feed-through matrix $D_{Q}$. This has the major advantage to prevent all direct effects on $r$ of the discontinuities in the scheduling signal $\rho$ (e.g., jumps due to the presence of the signum-function in (4)). The realization (11), with constant $A_{Q}$, also guarantees the robust stability of the filter. Moreover, it allows to obtain explicit discretization formulas for the matrices of an equivalent sampled-data model used for the on-board implementation of the filter (Varga et al., 2013).

The determination of $Q(s, \rho)$ is done using an approximated model of the actuator. Thus a perfect decoupling of the control input $u_{c}$ in the residual $r(t)$ can not be achieved from a practical point of view. A direct consequence is that high dynamic maneuvres may affect the residual $r(t)$, such that larger thresholds may be necessary to be used, and this also increases the minimum magnitude of detectable faults. This is why the need 
for an accurate approximation of the nonlinear actuator dynamics is instrumental for our approach. Furthermore, the determination of the least decision threshold guaranteeing the lack of false alarms is an important aspect, which is addressed in the next section.

Another related aspect is the presence in $\rho$ of uncertain components, say $\rho_{1}$, which are not measurable and therefore cannot be used as scheduling variables. Thus, instead $Q(s, \rho)$, only $Q\left(s, \rho_{2}\right)$ can be employed, where $\rho_{2}$ is formed from the rest of measurable components of $\rho$. Robust approximation methods suitable to determine $Q\left(s, \rho_{2}\right)$ from a given $Q(s, \rho)$ are discussed in (Varga, 2011). Once again, to guarantee the lack of false alarms in presence of the uncertainties $\rho_{1}$, special optimizationbased search techniques described in the next section need to be used to determine appropriate decision thresholds.

\subsection{Setup of residual evaluation and decision making blocks}

\subsubsection{Residual evaluation}

The evaluation of the residual signal often requires the computation of a measure of the residual signal energy, for which the 2-norm of the signal is usually an appropriate choice. For this purpose, for each fault type $j, j=1, \ldots, n_{f}$, a so-called Narendra signal evaluation scheme can be used of the form (Narendra and Balakrishnan, 1997)

$$
\theta_{r, j}(t)=\alpha_{r, j}|r(t)|+\beta_{r, j} \int_{0}^{t} e^{-\gamma_{r, j}(t-\tau)}|r(\tau)| d \tau,
$$

where $\theta_{r, j}(t)$ can be generated by the first order differential equation

$$
\begin{aligned}
\dot{\xi}_{j}(t) & =-\gamma_{r, j} \xi_{j}(t)+\beta_{r, j}|r(t)| \\
\theta_{r, j}(t) & =\xi_{j}(t)+\alpha_{r, j}|r(t)|,
\end{aligned}
$$

The filter parameters $\alpha_{r, j} \geq 0$ and $\beta_{r, j} \geq 0$ determine the firstorder filter nature (e.g., low pass, high pass, etc.) and have clear interpretations: $\alpha_{r, j}$ and $\beta_{r, j}$ are weights for instantaneous and long-term values, respectively, while $\gamma_{r, j}>0$ is the forgetting factor.

Choosing different filters for different types of fault is necessary because of different detection time requirements. For example, more critical faults as runaway or oscillatory failure require short detection times and therefore the corresponding residual evaluation schemes put more emphasis on current values than on time distant ones. On the contrary, the detection of less critical faults such as jamming or loss of efficiency is not time critical, and this is reflected in less stringent requirements on the detection times.

\subsubsection{Decision making}

In the simplest setup, the evaluation signal $\theta_{r, j}(t)$ is compared to a specific constant threshold $\tau_{j}$ in the decision making process to determine the decision signal $\iota(t)$ using the decision logic

$$
\begin{aligned}
& \theta_{r, j}(t)<\tau_{j} \Rightarrow \iota(t)=0 \Rightarrow \text { no fault } \\
& \theta_{r, j}(t) \geq \tau_{j} \Rightarrow \iota(t)=1 \Rightarrow \text { fault }
\end{aligned}
$$

The signal $\theta_{r, j}(t)$ is ideally equal to zero or sufficiently small in fault free situations, whereas it shall exceed the threshold $\tau_{j}$ when a fault occurs in the system. Hence, the appropriate selection of the values of the free parameters $\alpha_{r, j}, \beta_{r, j}$ or $\gamma_{r, j}$, together with an appropriate threshold $\tau_{j}$ essentially influences the performance of the FDD system.

\subsubsection{Optimal tuning of free parameters}

The free parameters $\alpha_{r, j}, \beta_{r, j}$ and $\gamma_{r, j}$ of the residual evaluation blocks and the threshold $\tau_{j}$ used in the decision blocks must be chosen to ensure that the requirements regarding typical performance criteria used in the industry such as the false alarm rate (FAR), the missed detection rate (MDR) or the detection time performance (DTP) are fulfilled (Goupil and Mar$\cos , 2012$ ). The simultaneous minimization of these quantities (e.g., by using multi-objective optimization techniques) would provide the best achievable detection performance. Unfortunately, analytical expressions of FAR and MDR are not available, and only surrogates can be used which are suitable only when FAR $=0$ and MDR $=0$ are imposed. The applicability of such surrogates based tuning has been demonstrated in (Varga et al., 2011b; Varga and Ossmann, 2012).

For an optimization based tuning setup, the requirements for the lack of false alarms and missed detections can be formulated as either optimization criteria or constraints. In the absence of faults, the requirement for no false alarms leads to a constraint on the false alarm bound

$$
\tau_{f, j}:=\sup _{f=0} \theta_{r, j}(t)<\tau_{j}
$$

where the supremum is taken for all admissible operation points, all relevant aircraft maneuvers, all admissible variations of uncertain parameters and for all relevant disturbances. The false alarm bound determines implicitly the least size $\|f\|$ of detectable faults, which satisfy

$$
\tau_{f, j}=\inf _{f \neq 0} \theta_{r, j}(t)
$$

where the infimum involves additionally all fault signals of a given type $j$.

The requirement for no missed detection can be also expressed as a constraint on the detection bound

$$
\tau_{d, j}:=\inf _{t \in\left[t_{f, j}, t_{\text {detec }, j}\right]} \theta_{r, j}(t) \geq \tau_{j},
$$

which must be satisfied for all relevant fault situations for the $j$-th type of fault. Here, $t_{\text {detec, } j}$ is the maximum admissible fault detection time and $t_{f, j}$ is the fault occurrence time. The concepts of false alarm bound, detection bound, least size detectable fault extends similar concepts introduced in (EmamiNaeini et al., 1988) for linear systems.

To ensure simultaneously the lack of false alarms and of missed detections, the condition $\tau_{f, j}<\tau_{d, j}$ must be fulfilled. If this condition is not fulfilled, then either the requirement for the lack of false alarms or the requirement for lack of missed detections can not be fulfilled. In practical applications, often the detection bound can be increased by relaxing the requirement on the least size of detectable faults. Alternatively, the false alarm bound can be decreased by constraining the size of 
admissible control signals. In this case, alarms resulted during maneuvers with large control inputs need further confirmation (see fault identification) using, for example, signal processing based signal classification techniques.

Finally, let $t_{d, j}$ be the fault detection time defined as the least value of time $t$ for which $\theta_{r, j}(t) \geq \tau_{j}$. Then, the constraint on detection times can be expressed as

$$
t_{d, j} \leq t_{\text {detec }, j}
$$

or equivalently in a normalized form

$$
D T P:=\frac{t_{d, j}-t_{f, j}}{t_{d e t e c, j}-t_{f, j}}<1 .
$$

The tuning approach assumes that the FDD system is running jointly with a robustly stable closed-loop system as shown in Fig. 1. For each type of fault $j$, the value of the threshold $\tau_{j}>0$ can be chosen arbitrarily, while the free parameters $w_{j}:=\left(\alpha_{r, j}, \beta_{r, j}, \gamma_{r, j}\right)$ can be determined by solving suitable optimization problems:

1. Feasibility problem: Determine $w_{j}$ such that

$$
\tau_{f, j}<\tau_{j}, \quad \tau_{d, j} \geq \tau_{j}, \quad t_{d, j} \leq t_{d e t e c, j}
$$

2. Optimal detection time performance: Determine $w_{j}$ which minimizes $t_{d, j}$ subject to

$$
\tau_{f, j}<\tau_{j}, \quad \tau_{d, j} \geq \tau_{j},
$$

3. Multi-objective minimization problem: Determine $w_{j}$ which simultaneously minimizes $\tau_{f, j}$ and $-\tau_{d, j}$, subject to $t_{d, j} \leq t_{\text {detec }, j}$.

4. Optimal robustness margin: Determine $w_{j}$ which maximizes $\tau_{d, j}-\tau_{f, j}$ or minimize $\tau_{f, j} / \tau_{d, j}$ subject to $t_{d, j} \leq$ $t_{\text {detec }, j}$.

If any of the first two problems is solvable, then it is guaranteed that no false alarms and no missed detection can occur.

The solution of each of above problems involves solving optimization problems with semi-infinite (parameter dependent) constraints. Each evaluation of constraints involves solving global optimization problems over function spaces, thus these problems are computationally intractable. To compute suboptimal solutions, appropriate relaxation techniques can be used, as the discretization of the uncertain parameter search space (e.g., via gridding or multi-models) and using predefined finite set of relevant maneuvers, wind disturbances, sensor noise levels and faults. Although the number of optimization parameters is small, even with the above simplifications a substantial computational effort is necessary, because all function evaluations involve nonlinear system simulations and global optimizations over the uncertain parameter space. Parallel computation techniques for global optimizations can significantly reduce computational times.

\subsection{Fault identification}

In this section the fault identification aspects related to several types of actuator faults are considered. The fault identification block for the $j$-th fault type generates the confirmation signal $\eta_{j}(t)=1$, if the fault is confirmed or $\eta_{j}(t)=0$, otherwise. The fault identification is only triggered, at a time instant say $t_{d, j}$, when the presence of a fault has been detected (i.e., the output of the decision block in Fig. 2 is $\iota_{j}(t)=1$ for $\left.t \geq t_{d, j}\right)$. This separation between fault detection and fault identification is a distinctive feature of our FDD approach, when compared with other techniques as those used in (Goupil, 2010; Gheorghe et al., 2013), where the fault identification algorithms must run permanently on the on-board flight control computers.

To address the fault identification aspects, signal processing based techniques can be used, where for each type of fault specific methods are devised. Suitable methods for identification of several classes of additive faults (jamming, oscillatory failure, runaway and loss of efficiency) are described in this section. These techniques are employed to design a complete FDD system for monitoring actuator faults as described in Section 4. To simplify the notation in the rest of the paper, the fault evaluation and decision related variables (e.g., $\left.\theta(t), \eta(t), t_{d}, \iota(t)\right)$ are used without the index $j$ when discussing particular types of faults.

\subsubsection{Identification of jamming}

The jamming (or lock-in-place failure) of an aircraft control surface creates a dissymmetry in the aircraft configuration, which must be compensated by appropriate deflections of other control surfaces. Therefore, the jamming leads to the degradation of the aircraft performance due to the increased drag, which depends on the amplitude and localization of the failure. For example, during a long time aircraft operation, a surface jamming may produce substantial drag which can lead to excessive fuel consumptions (with all associated negative environmental effects) and can even impede the fulfillment of the flight mission (i.e., the need for landing on a diverting airport for refueling). Therefore, the timely detection and identification of jamming, especially of the primary control surfaces (e.g., elevator, ruder, ailerons), is important for both an economical and easy-to-handle operation of an aircraft.

To confirm jamming at a constant deflection $u_{\text {jam }}$, the following computations are needed:

1. Collect $n$ samples of the output variable $u(t)$ :

$$
u_{1}=u\left(t_{d}+T\right), \ldots, u_{n}=u\left(t_{d}+n T\right) \text {. }
$$

2. Compute the mean: $\bar{u}:=\frac{1}{n} \sum_{i=1}^{n} u_{i}$.

3. Compute the variance: $\sigma_{u}:=\frac{1}{n-1} \sum_{i=1}^{n}\left(u_{i}-\bar{u}\right)^{2}$.

4. If $\sigma_{u} \leq \tau_{u}$, then set $\eta(t)=1$; else, set $\eta(t)=0$.

Here, $T$ is the sampling period used to collect the $n$ samples and $\tau_{u}$ is a threshold for zero (or negligible) variance. Typical values of the main parameters of the identification procedure are $T=0.01, n=50 \div 300$ and $\tau_{u}=0.01 \div 0.1$.

This computational approach requires the storage of $n$ samples, which is not desirable for on-board computations. Fortunately, it is possible to use recursive evaluation formulas of the mean and sample variance which avoid any additional storage needs and are based on a numerically stable computational 
method (Welford, 1962). The implementation of this algorithm starts with the initializations $m_{1}=u_{1}$ and $s_{1}=0$, and recursively computes

$$
\begin{aligned}
m_{k} & =m_{k-1}+\left(u_{k}-m_{k-1}\right) / k \\
s_{k} & =s_{k-1}+\left(u_{k}-m_{k-1}\right)\left(u_{k}-m_{k}\right)
\end{aligned}
$$

for $k=2, \ldots, n$. Then, $u_{\text {jam }}=\bar{u}=m_{n}$ and $\sigma_{u}=s_{n} /(n-1)$. With this approach, the confirmation time $t_{c}$ of a surface jamming is roughly equal to $t_{c}=t_{d}+n T$.

Surface sensor bias (called also liquid jamming) can be easily identified, by applying the same signal processing algorithm to the residual signal $r(t)$ as that applied to $u(t)$. The resulting bias is the nonzero mean value $r_{j a m}=\bar{r}$, while the covariance $\sigma_{r}$ serves for the confirmation of the presence of the bias.

The special case of jamming of a control surface at null deflection can alternatively be handled by computing the evaluation signals $\theta_{u_{c}}$ and $\theta_{u}$

$$
\begin{aligned}
& \theta_{u_{c}}(t)=\int_{0}^{t} e^{-\gamma_{u_{c}}(t-\tau)}\left|u_{c}(\tau)\right| d \tau \\
& \theta_{u}(t)=\int_{0}^{t} e^{-\gamma_{u}(t-\tau)}|u(\tau)| d \tau
\end{aligned}
$$

where $\theta_{u_{c}}(t)$ and $\theta_{u}(t)$ can be generated by first order differential equations similar to that in (14). The jamming at null deflection can be confirmed if the conditions for non-zero input $\theta_{u_{c}} \geq \tau_{u_{c}}$ and zero output $\theta_{u}<\tau_{u}$ are simultaneously fulfilled, where $\tau_{u_{c}}$ and $\tau_{u}$ are appropriate thresholds for nonzero and zero energy signals, respectively. A typical setting of the corresponding forgetting factors $\gamma_{u_{c}}$ and $\gamma_{u}$ must fulfill the condition $\gamma_{u}<\gamma_{u_{c}}$ imposed by the signal transmission causality requirements. This fault identification scheme significantly enhances the overall robustness of the FDD system.

The alternative approach for the identification of jamming (and also of runaway) used in (Gheorghe et al., 2013) represents an enhancement of the current industrial practice by filtering (with a low pass filter) of a particular residual evaluation signal to attenuate the influence of measurement noises. The goal of this approach is to allow the choice of smaller decision thresholds, which permit the identification of jamming at smaller surface deflections.

\subsubsection{Identification of oscillatory failure cases}

Two types of oscillatory failure cases (OFCs) are usually considered. The so-called liquid failure is an additive oscillatory fault signal inside the actuator positioning control loop. The solid failure involves an oscillatory signal which completely replaces a normal signal in the actuator positioning loop. According to Goupil (2010), the relevant frequency range for the OFC is $0.1-10 \mathrm{~Hz}$. The fault identification challenge for OFC is the need of an early detection, which imposes short detection times corresponding to a few oscillation periods. Another challenge is the detection of small amplitude oscillations of the control surfaces in the presence of measurement noise. Finally, the fault detection and identification performance must be robustly achieved over the whole flight envelope, for various pilot maneuvers and wind conditions, and over the whole range of uncertain parameter variations. The early detection of an OFC is important to prevent high loads and for taking into account stringent structural design objectives (Goupil, 2010; Alcorta-Garcia et al., 2011).

By using a sufficiently accurate LPV approximation (1) of the actuator nonlinear model (2) as basis for residual generator synthesis, the resulting residual signal $r$ will contain the same oscillatory components as the fault signal. Once the occurrence of a fault has been detected using the decision logic (15), the fault identification stage follows aiming to detect the presence of oscillations in $r$ in the relevant frequency band. For the identification of OFC, a signal processing based technique has been proposed by Goupil (2010), which involves sub-band filtering of the residual signal followed by an oscillation counting. Separate schemes are used to identify liquid and solid type OFCs. In this section, an alternative approach is described, which is based on recursive Fourier analysis techniques, and thus is easily implementable on-board. Furthermore, the proposed approach needs no special treatment of different types of OFCs.

A rigorous approach to identify oscillations in noise corrupted signals is the periodogram method (Stoica and Moses, 1997), which is based on determining the power spectrum of a signal using the discrete Fourier transform (DFT). The DFT is easily computable using the fast Fourier transform (FFT) algorithm and allows a satisfactory accurate evaluation of the oscillation frequency together with strong statistical guarantee of the presence of the oscillatory signal. Still, the on-board implementation of FFT-based frequency analysis is questionable, due to the strict code certification requirements.

To overcome these limitations, a recursive version of the DFT, as described in (Morelli, 2000), can be used to detect oscillations in real-time. Let $T$ be the sampling period, $t_{d}$ the fault detection time and let $n$ be the expected length of the time series $r\left(t_{i}\right)$, for $t_{i}=t_{d}+i T$, for $i=0,1, \ldots, n-1$. The DFT computes

$$
X(\omega):=\sum_{i=0}^{n-1} r\left(t_{i}\right) e^{-j \omega t_{i}}
$$

for a given frequency $\omega$. The computation of $X(\omega)$ can be done recursively by defining the partial sum

$$
Y_{k}(\omega)=\sum_{i=0}^{k} r\left(t_{i}\right) e^{-j \omega t_{i}}
$$

and observing that

$$
Y_{k}(\omega)=Y_{k-1}(\omega)+r\left(t_{k}\right) e^{-j \omega t_{k}}
$$

for $k=1, \ldots, n$. Evidently, $X(\omega)=Y_{n-1}(\omega)$, where the iteration (22) is initialized with $Y_{0}(\omega)=0$. An oscillation of frequency nearby to $\omega$ is detected at iteration $k=k_{\text {detec }}$ if $\left|Y_{k_{\text {detec }}}(\omega)\right|>$ $\tau_{\text {freq }}$, where $\tau_{\text {freq }}$ is a suitable threshold. The main appeal of this approach is that usually $k_{\text {detec }} \ll n$, thus fast detection of the presence of oscillations nearby a frequency $\omega$ is possible.

For the choice of the threshold $\tau_{\text {freq }}$, the expression $\tau_{\text {freq }}=$ $\frac{n}{2} \tau_{0}$ can be employed, where $\tau_{0}<1$ is a normalized threshold for unit amplitude sinusoidal signals of frequency $\omega$. The value 
of $\tau_{0}$ generally accounts for the need to avoid undetected oscillations of frequency $\omega_{x}$ nearby $\omega$. Recall that the frequency resolution of the DFT for $n$ samples and a sampling period $T$ is $\frac{2 \pi}{T n}$, and therefore the frequency bin around $\omega$ contains all frequencies $\omega_{x}$ such that $\left|\omega_{x}-\omega\right| \leq \frac{\pi}{T n}$. The magnitude of the resulting Fourier coefficient for a unit amplitude sinusoidal signal of frequency $\omega_{x}$ is

$$
|X(\omega)|=\frac{1}{2}\left|\frac{\sin \left[\left(\omega_{x}-\omega\right) n T / 2\right]}{\sin \left[\left(\omega_{x}-\omega\right) T / 2\right]}\right| \leq \frac{n}{2}
$$

The maximum magnitude drop within a frequency bin results for $\omega_{x}=\omega \pm \frac{\pi}{T n}$ as

$$
|\underline{X}(\omega)|=\frac{1}{2} \frac{1}{\left|\sin \frac{\pi}{2 n}\right|}
$$

Since $|\underline{X}(\omega)| /(n / 2)>2 / \pi$ for all $n, \tau_{0}=2 / \pi$ can be taken as a safe threshold for each frequency bin.

Using the above choice for $\tau_{0}$, the minimum number of frequency values such that the union of the associated frequency bins covers the relevant frequency domain can be easily determined. Let $\Omega=\left\{\omega_{1}, \omega_{2}, \ldots, \omega_{m}\right\}$ be the frequency grid to be chosen with $\omega_{1}>\omega_{2}>\ldots>\omega_{m}$. Each $\omega_{\ell}$ is the midpoint of an interval $\mathcal{I}\left(\omega_{\ell}\right):=\left[\underline{\omega}_{\ell}, \bar{\omega}_{\ell}\right]$, which defines a frequency bin with a resolution of $\frac{2 \pi}{n\left(\omega_{\ell}\right) T}$, where $n\left(\omega_{\ell}\right)$ is the corresponding number of samples (to be chosen). The choice of these intervals must ensure that their union $\cup_{i=1}^{m} \mathcal{I}\left(\omega_{\ell}\right)$ fully covers the relevant frequency domain for the OFC. A non-overlapping choice is always possible by imposing $\bar{\omega}_{\ell}=\underline{\omega}_{\ell-1}$, and results by choosing $\underline{\omega_{\ell}}$ for $\ell=1,2, \ldots, m$ according to

$$
\underline{\omega}_{\ell}=\frac{\underline{\omega}_{\ell-1}}{2}
$$

where $\underline{\omega}_{0}$ is the maximum frequency in the relevant frequency domain. $n\left(\omega_{\ell}\right)$ can be chosen to satisfy the worst-case detection time requirement of two periods for the maximum frequency in the $\ell$-th bin $\bar{\omega}_{\ell}=\underline{\omega}_{\ell-1}$, that is, $n\left(\omega_{\ell}\right) T=4 \pi / \underline{\omega}_{\ell-1}$. For this choice, taking into account (23), the simple relations $n\left(\omega_{\ell}\right)=2 n\left(\omega_{\ell-1}\right)$ between two successive numbers of samples hold. For example, using the above approach, the frequency domain $[0.15,10] \mathrm{Hz}$ can be covered using a grid of $m=6$ frequencies

$$
\Omega=2 \pi \times\{7.5,3.75,1.875,0.9375,0.4687,0.2343\}
$$

and the corresponding values $\{20,40,80,160,320,640\}$ of $n\left(\omega_{\ell}\right)$ for a typical sampling period of $T=0.01 \mathrm{~s}$.

The recursive algorithm (22) can be implemented efficiently and thus is appealing for real-time applications. Since usually $m \ll n$, the computations for $n-m$ frequency values of no interest can be discarded (which would be automatically produced when employing the FFT). For increased efficiency, the precomputed quantities $W(\omega):=e^{-j \omega T}$ for each $\omega \in \Omega$ can be used and the relations

$$
s_{k}:=e^{-j \omega t_{k}}=W(\omega) e^{-j \omega t_{k-1}}=W(\omega) s_{k-1}
$$

can be exploited. Thus, at each step $k>2$ the quantities

$$
\begin{aligned}
s_{k} & =W(\omega) s_{k-1} \\
Y_{k}(\omega) & =Y_{k-1}(\omega)+r\left(t_{k}\right) s_{k}
\end{aligned}
$$

have to be computed for each $\omega \in \Omega$. These iterations are initialized with $Y_{1}(\omega)=r\left(t_{0}\right)$ and $s_{1}=1$. Taking into account that the intervening $r\left(t_{k}\right)$ in (22) is a real quantity, the number of floating point operations (flops) to compute $Y_{k}(\omega)$ and update $s_{k}$ is 1 complex and 2 real multiplications and 1 complex addition (equivalent to 6 real multiplications and 2 real additions). Thus, the operations at each step involve $8 m$ flops. Finally, the iteration is stopped if an oscillation has been detected at a certain iteration $k_{\text {detec }}$. The total number of operations is about $8 m\left(k_{\text {detec }}-1\right)$ flops.

\subsubsection{Identification of runaway}

An actuator runaway (or hardover) takes place when a large, uncommanded surface deflection occurs and the surface tends to lock in its extreme position. The transition to this position may occur with arbitrary variation rates (constant or timevarying). In the case of a runaway, excessive structural loads can be expected, and therefore it must be very quickly detected and identified by the FDD system, before its full development. For example, the fault detection and identification times can be related to a certain allowed maximum variation of the surface deflection angle.

The early detection and identification of runaway can be done by checking that the surface deflection variation rate $\dot{u}$ is above or below a certain allowed slew rate $\dot{u}_{\max }$ or $-\dot{u}_{\text {max }}$, respectively,

$$
|\overline{\dot{u}}| \geq \dot{u}_{\max }
$$

where $\overline{\dot{u}}$ is the mean value of the derivative over a given time period. The computation of an approximation of this mean can be done recursively as

$$
\overline{\dot{u}} \approx \frac{u\left(t_{i}\right)-u\left(t_{d}\right)}{t_{i}-t_{d}}
$$

where $t_{d}$ is the detection time and $t_{i}=t_{d}+i T, i=1,2, \ldots$, with $T$ a suitable sampling period.

Using this approach, large surface deflections in relatively short times will be always recognized as runaways, as for example, an oscillatory failure with a large oscillation amplitude. For practical purposes this is perfectly acceptable, since the only meaningful handling in such cases is the disconnection of the faulty actuator. However, to ensure the identifiability of oscillatory failures with small amplitudes, the confirmation of runaway should be done only if the amplitude of the residual signal is above a certain maximum value $\tau_{\text {rmax }}$

$$
|r(t)| \geq \tau_{\text {rmax }}
$$

While this works satisfactorily for liquid type OFCs, still solid type OFCs can be occasionally diagnosed as runaways. 


\subsubsection{Identification of loss of efficiency}

Several types of fault can be categorized as loss of efficiency faults, as for example, speeding-up of the actuator dynamics due to a disconnection (e.g., broken rod), slowing-down of the actuator dynamics due to a reduction of the actuator gain, or the inability to reach a commanded deflection (e.g., due to loss of power or a sensor calibration error). Since the first two types of faults are parametric faults (i.e., changes in the actuator gains), they can not be easily detected using the additive faults based approach. This is why, only the third category of fault is considered which, for simplicity, will be called loss of efficiency fault. This fault can be easily detected as additive fault using the proposed fault detection approach. In what follows the identification of this type of fault using signal processing based techniques is discussed.

Starting from the detailed actuator description (2), the assumed behavior during the fault is described by

$$
\dot{\tilde{u}}=K(p, \tilde{u}, \dot{\tilde{u}})\left(v u_{c}-\tilde{u}\right),
$$

where $0<v<1$ is a constant parameter which describes the loss of efficiency of the actuator. See (Bošković et al., 2005) for a similar fault modelling approach. An accurate estimate of the fault free output $u$ of the actuator can be generated using the LPV model (1). It follows that the outputs of the faulty actuator $\tilde{u}(t)$ and of the fault free actuator model $u(t)$ are related at all time moments simply as $\tilde{u}(t)=v u(t)$. The validity of this relation can be easily checked in real-time as follows.

First $n$ samples of the time-varying signal $v(t):=u(t) / \tilde{u}(t)$ are computed as

$$
v_{i}:=\frac{u\left(t_{i}\right)}{\tilde{u}\left(t_{i}\right)} .
$$

where $t_{i}=t_{d}+i T$, for $i=0, \ldots, n-1$ with $T$ a suitable sampling period. The confirmation of the efficiency loss failure requires a constant value of $v(t)$. Therefore, the mean $\bar{v}$ and sample variance $\sigma_{v}$ of $v$ are computed using the recursive algorithm in (20), with $u_{k}$ replaced by $v_{k}$. Then, the efficiency loss failure is confirmed if $\sigma_{v} \leq \tau_{\sigma_{v}}$, where $\tau_{\sigma_{v}}$ is a suitable threshold for zero variance.

\subsection{Robustness assessment}

The assessment of the robustness of the FDD system serves primarily to check the performance of the FDD system in presence of all relevant uncertainties (parameters, operation points, maneuvers, disturbances, noise). It is also part of the verification and validation $(\mathrm{V} \& \mathrm{~V})$ of the aircraft design and of the aircraft certification (clearance). By discovering possible hidden design weaknesses and by finding worst-case parameter combinations, robustness assessment is also a valuable design aid.

The robustness assessment, due to the multitude of performed tests, is computationally expensive. Significant cost reduction can be achieved by using efficient assessment techniques, which may contribute to significantly reduce the development costs of aircraft monitoring systems. The industrial standard is the use of Monte Carlo (MC) simulation and parameter gridding-based techniques for this purpose. However, several recent European projects (Fielding et al., 2002; Varga et al., 2011a) demonstrated the usefulness of clearance methods using advanced robustness analysis methods based on worst-case (WC) search with global optimization techniques. The usefulness of these methods for the V\&V of FDD systems is shown in what follows.

The Monte Carlo simulation and parameter gridding based methods have the main advantage of being simultaneously applicable to several performance criteria. Also the computed results are widely accepted for industrial V\&V. However, these methods only single out parameter combinations which violate some design requirements, and thus produce only a rough estimation of worst-case parameter combinations. Also, because of the discrete character of the search, both methods can fail in finding violation even if they exist, if these parameter combinations lie in intermediary points (which are not tested).

Within the ADDSAFE Project, the Functional Engineering Simulator (FES) environment described in (Goupil and Marcos, 2012) has been used for the verification of the FDD designs. The FES is based on MATLAB-Simulink and built around the aircraft closed-loop nonlinear simulation benchmark model augmented with the designed FDD system as shown in Fig. 1. For randomly generated combinations of the uncertain parameters, the analysis tools implemented in FES evaluate several indicators which allow to assess the performance characteristics of the FDD system.

While Monte Carlo simulation can be seen as a useful verification technique (e.g., well suited for rapid prototyping), for validation purposes it is necessary to use alternative approaches which are able to cover continuous uncertain parameter ranges, provide guarantees for lack of hidden weaknesses and determine worst-case parameter combinations to serve for design enhancements. Therefore, for the validation of the FDD design, a global optimization-based worst-case search is a suitable approach which fulfils all the above requirements and delivers (usually in shorter times than with Monte Carlo simulation) accurate estimations of worst-case parameter combinations, for example, by maximizing the fault detection and identification time. The optimization environment MOPS (Multi-Objective Parameter Search) of DLR (Joos, 1999) provides an easy access to different global optimization algorithms together with visualization tools of the computed results and even of the intermediary iterations. For the maximization of the fault detection and identification time, the differential evolution (DE) global search method has been employed (Storn and Price, 1997). This method allows to perform many function evaluations in parallel, which significantly contributes to alleviate the associated computational burden due to expensive simulation runs.

The FDD system synthesis procedure relies on extensive tuning, which usually allows to avoid false alarms and missed detection. Therefore, only the application of the worst-case search based approach to determine the parameter combinations leading to the worst detection time performance is necessary. Thus the robustness analysis tries to find worst case combinations of uncertain parameters, aircraft maneuvers, wind and noise inputs which maximizes the DTP defined in (19), where $t_{d}$ is the total time necessary for fault detection and identification. It is to be expected that the largest DTP provided by the worst-case search 
is larger than the one produced by Monte Carlo or griding based techniques.

\section{Synthesis of a robust FDD system}

In this section we apply the proposed synthesis methodology for the design of a robust FDD system for monitoring $n_{f}=4$ categories of additive flight actuator faults, namely the jamming, oscillatory failure, runaway and loss of efficiency, in the case of a civil aircraft elevator. The employed FDD system architecture is depicted in Fig. 2.

The scheduling variable has been defined as $\rho=$ $(u, \operatorname{sign}(\dot{u}), p)$, where the components of the vector $p$ are the calibrated airspeed $V_{\text {cas }}$, the aircraft altitude $h$, the aircraft mass $m$ and the position of the center of gravity $X_{c g}$ along the $x$-axis. The common LPV residual generator has the input-output form (8) with the state space realization (11) and the state space matrices given in (12). The typical values for the filter pole $a=14$ and actuator gain $k_{0}=14$ have been employed. The implicit assumption in this paper is that no (sensor) faults occur when measuring the scheduling variables. In the case of detected faulty measurements, simpler approximations of the gain $k(\rho)$ can be employed (e.g., even a constant gain), which however may lead to performance diminutions.

In what follows we describe the application of the specific methodological steps for the monitoring of each individual fault. In all cases simulation-based robustness assessment results are presented, which illustrate the robust performance of the resulting FDD system. The main uncertainties considered in the robustness analysis are the flight conditions (altitude, speed) over the whole flight envelope and the full range of variations of the aircraft mass and center of gravity position. While the limits of the altitude $h$ and the mass $m$ are fixed, the limits of the center of gravity $X_{c g}$ depend on the actual mass, while the limits of the calibrated airspeed $V_{c a s}$ depend on the actual mass and altitude. This leads to somewhat reduced bounds for these parameters depending on the concrete flight condition (Varga et al., 2011b). Additionally, uncertainties up to 5\% in the aerodynamic database, up to $10 \%$ in the measurements of altitude and velocity, and up to $10 \%$ in the accuracy of the estimations of the mass and center of gravity have been considered.

\subsection{Monitoring control surface jamming}

\subsubsection{Parameter tuning}

The free parameters of the fault evaluation and decision block can be determined in an optimal way, such that typical requirements as lack of false alarms and missed detections, as well as constraints on detection times are fulfilled. Multi-objective optimization based tuning strategies have been described, for example, in (Varga and Ossmann, 2012), where the term integrated tuning has been employed to emphasize that the FDD system runs with a robustly stable closed-loop system as shown in Fig. 1.

For the tuning of the FDD system, the values of the parameters $\alpha_{r}, \beta_{r}$ and $\gamma_{r}$ of the residual evaluation block have been determined by maximizing the gap $\tau_{d}-\tau_{f}$, where $\tau_{f}$ is the false alarm bound in (16) and $\tau_{d}$ is the detection bound in (17). The maximization of $\tau_{d}-\tau_{f}$ has been done such that the fault detection time satisfies $t_{d} \leq t_{\text {detec }}$ in (18).

The optimal setting of the parameter of the residual evaluation filter (13) is $\alpha_{r}=0.3, \beta_{r}=1$ and $\gamma_{r}=0.2$, while the choice $\tau=0.1$ of the detection threshold satisfies $\tau_{f}<\tau \leq \tau_{d}$. This leads to a completely satisfactory fault detection performance.

\subsubsection{Verification and validation results}

The determination of the threshold $\tau$ relies on worst-case search-based evaluations of the false alarm and detection bounds, and therefore its choice above guarantees the fulfillment of all basic performance requirements. To provide supplementary information on the robustness of the overall detection time performance of the FDD system, optimization-based search techniques have been used to determine the global worstcase detection time. The analysis itself relies on repeated simulations of the nonlinear model of the closed-loop aircraft including a nonlinear control law ensuring robust stability over the whole flight envelope (see Fig. 1).

A Monte Carlo simulations based validation campaign with FES covered a total of 1200 points in the flight envelope, which have been used to test the robustness of the designed FDD system in fault free cases during six demanding maneuvers (e.g., pitch protection and angle of attack protection maneuvers). None of these test points/maneuvers triggered any false alarms. To test the detection performance, the jamming at small deflections of the elevator during cruise and smooth turn maneuvers has been tested in 1000 different flight points. In particular, jamming at null deflection occurring in cruise has been detected during turn maneuvers. Note that jamming at small deflections during smooth maneuvers poses challenges for the fault detection due to the small amplitudes of the residual signals. The Monte Carlo analysis provided a mean detection and identification time of $3.57 \mathrm{sec}$ with a standard deviation of $0.92 \mathrm{sec}$ and a maximum detection time of $10.65 \mathrm{sec}$. In Fig. 3 the histogram of the identification times determined by the Monte Carlo simulations are depicted. It can be observed that most of the 1200 fault cases are detected and identified nearly in minimum time of $3.01 \mathrm{sec}$, and only a few ones are delayed. These delays are caused by the presence of very small control inputs and hence, small residuals, especially at high velocities in the flight envelope.

The optimization-based worst-case search has been performed on a Linux cluster with $16 \mathrm{CPUs}$ running at $2.6 \mathrm{GHz}$ the MATLAB Parallel Toolbox. An optimization run, involving about 1500 function evaluations, took around 15 minutes. The resulting worst case detection and identification time of the jamming for the designed FDD system was $18.09 \mathrm{sec}$, which is still completely satisfactory for an industrial usage. Note that this time is about $40 \%$ higher than $10.65 \mathrm{sec}$, the time found by Monte Carlo simulations. Fig. 4 depicts the search points in the normalized flight envelope and normalized weight-andbalance diagram, which have been generated by the DE method during the worst case optimization. Values of the identification time larger than $10 \mathrm{sec}$ are marked with red crosses, while values lower than $10 \mathrm{sec}$ are marked with blue circles. As it can 


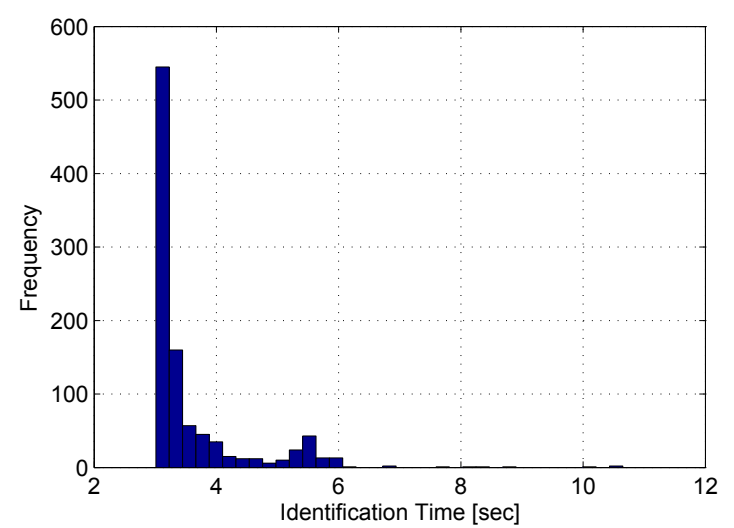

Figure 3: Histogram of the fault identification times

be seen, the more critical region of the flight envelope from the point of view of a fast identification of the jamming corresponds to high velocities in combination with a more forward center of gravity position. This parameter combination makes the control task easier by needing smaller size inputs to steer the aircraft, but adversely affects the detection and identification times of the faults.
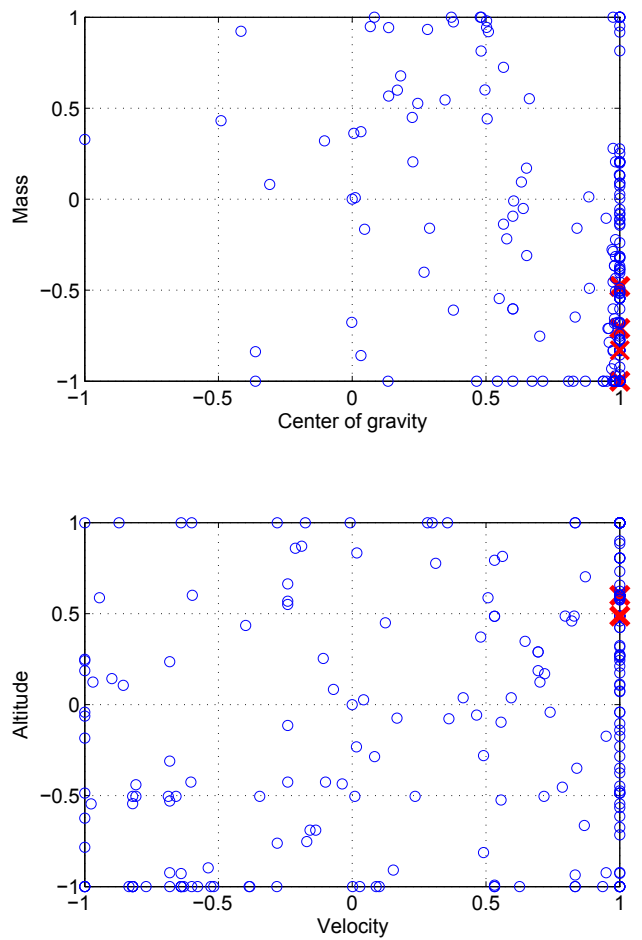

Figure 4: Search points generated by the DE method

\subsubsection{Industrial evaluation}

The industrial evaluation has been performed using hardware-in-the-loop simulations with a flight actuator test bench built around a real elevator actuator with simulated command inputs, aerodynamic forces and hydraulic pressures, providing continuous monitoring of computer internal variables. This bench offers the possibility to validate the FDD system in degraded configurations, as in the case of low hydraulic pressure and high loads on the control surface. The industrial validation campaign consisted of the assessment of the robustness (i.e., the lack of false alarms) and of the detection performance (i.e., the lack of missed detections and satisfactory detection time). The robustness of the FDD designs has been assessed during pure lateral maneuvres, pure longitudinal maneuvres and during mixed maneuvres (combining lateral and longitudinal movement in the same maneuvres). Both smooth and dynamic maneuvres have been performed, as for example, auto-pilot maneuvres, flight control checks, take-off and landing, certification maneuvres, etc. The detection performances have been assessed by simulating the jamming failure scenario, during a classical flight and during specific maneuvres. The results have shown a high degree of robustness of the designed FDD system for the whole range of tests and a highly satisfactory detection performance. Further details on the industrial setup can be found in (Varga et al., 2013).

A typical detection result of a jamming occurring at $t_{f}=6 \mathrm{~s}$ at a surface deflection of $-2^{\circ}$ is presented in Fig. 5 .
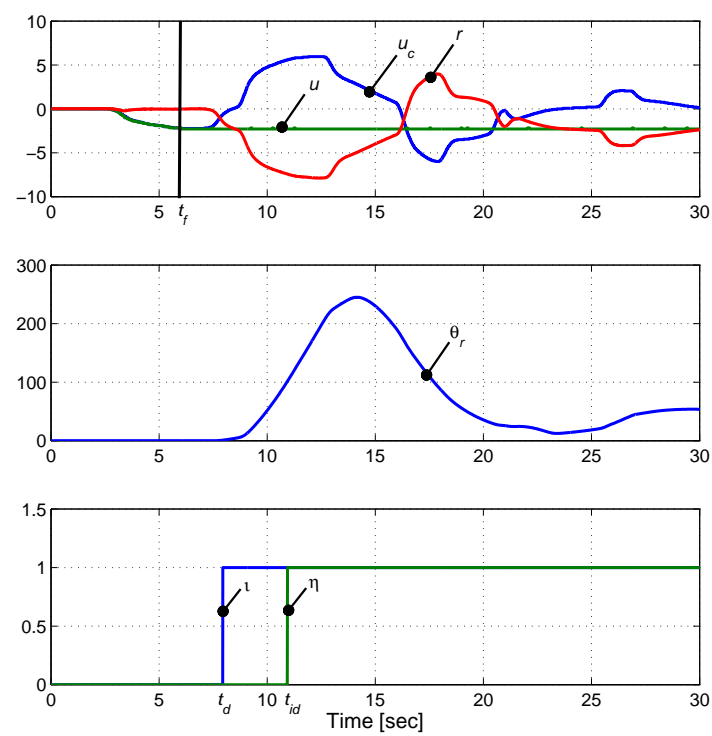

Figure 5: Detection and identification of a jamming at $-2^{\circ}$

In Fig. 6 an example is presented for the detection of a jamming at a null surface deflection at $t_{f}=0 \mathrm{~s}$. As it can be observed, the dedicated identification scheme based on Narendratype filters (21) has a significantly shorter identification time $t_{i d 0}$ than the identification time $t_{i d}$ resulting when employing a general purpose fault identification method of jamming at arbitrary deflections.

\subsection{Monitoring of $\mathrm{OFC}$}

\subsubsection{Parameter tuning}

For the tuning of the FDD system, the values of the parameters $\left(\alpha_{r}, \beta_{r}, \gamma_{r}\right)$ of the residual evaluation block and the threshold $\tau$ of the decision making block have been determined by 

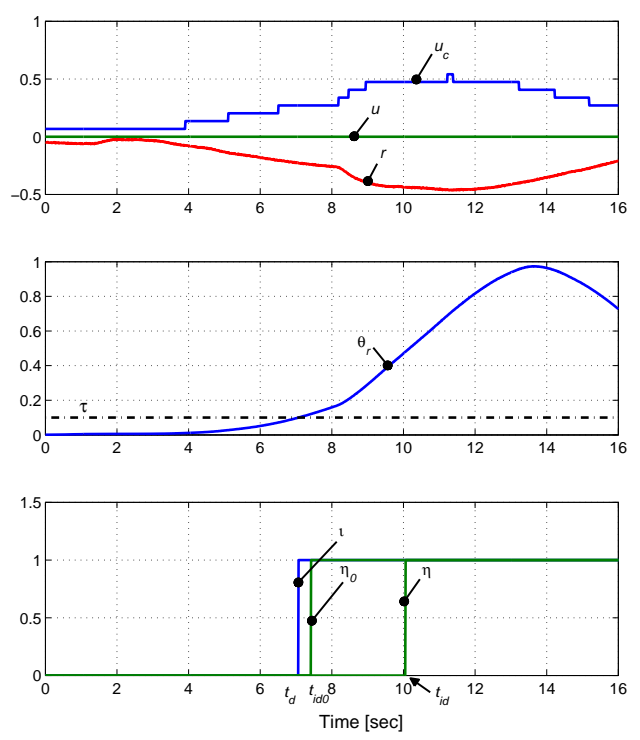

Figure 6: Detection and identification of a jamming at $0^{\circ}$

maximizing the detection gap $\tau_{d}-\tau_{f}$. The optimal setting with $\alpha_{r}=0.85, \beta_{r}=0.8, \gamma_{r}=0.08$ and the choice $\tau=0.7$ led to a completely satisfactory fault detection performance. Recall that the signal processing based identification of the OFC frequency presented in the subsection 3.4.2 is only triggered if the evaluation signal $\theta_{r}(t)$ crosses the corresponding threshold $\tau$.

\subsubsection{Verification and validation results}

The robustness of the designed FDD system has been thoroughly tested for both fault free and faulty situations in the whole flight envelope and full range of aircraft parameter variations. To check for the lack of false alarms, typical maneuvers as for example, piloted flights with various pilot inputs (longitudinal/lateral stick doublets, pedal input demand, nose up/nose down demands) or typical navigation maneuvers (level flight, flight path angle target mode, yaw angle target mode, speed change, steady sideslip, coordinated turn, etc.) have been used.

Using optimization-based worst-case search, the normalized detection time DTP defined in (19) has been used as clearance criterion, where $t_{f}$ is the time of occurrence of the fault, $t_{d}$ is replaced by $t_{i d}$ which denotes the time of the identification of OFC and $t_{\text {detec }}$ is the maximum admissible value for $t_{i d}$. A maximum value above 1 of this criterion indicates a violation of the required detection time performance.

A typical fault-free maneuver is depicted in Fig. 7, where the actuator output $u$ follows almost instantly the demanded signal $u_{c}$. The generated residual $r$ is corrupted only by the measurement noise in $u$, which is also present in the evaluation signal $\theta_{r}$. This however remains below the threshold $\tau$, indicating no fault.

Fig. 8 illustrates the occurrence of a liquid OFC with a frequency of $0.5 \mathrm{~Hz}$ (i.e., $\omega=\pi$ ) in the actuator output $u$ at $t_{f}$. The visible difference between $u_{c}$ and $u$ indicates that the residual signal has likely an oscillatory behavior. Indeed, the evalua-

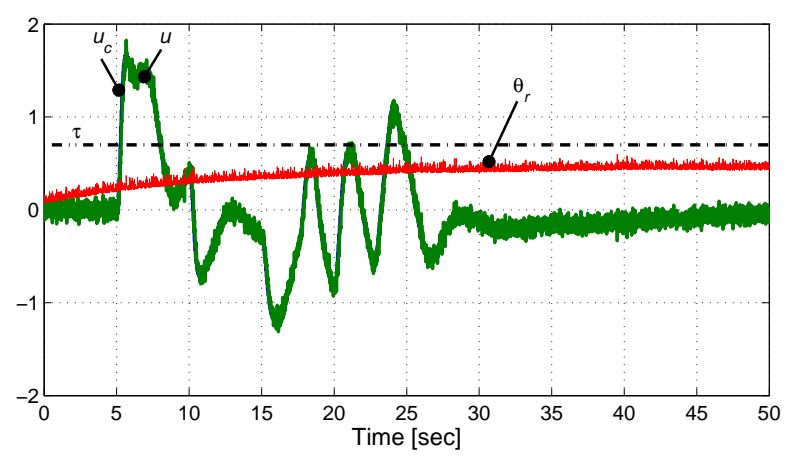

Figure 7: Example for fault free maneuvering

tion signal $\theta_{r}$ increases rapidly and crosses the threshold $\tau$ at $t_{d}$, when the fault is detected. The signal based fault identification indicates at $t_{i d}$ that the value $\left|Y_{k}(\pi)\right|$ of the power spectral density of the residual becomes larger than the threshold $\tau_{\text {freq }}$. In this example the total detection time of the OFC is $t_{i d}-t_{f}=2.6 \mathrm{sec}$, of which $t_{d}-t_{f}=0.4 \mathrm{sec}$ are required to detect the occurrence of a fault and $t_{i d}-t_{d}=2.2 \mathrm{sec}$ to identify it.
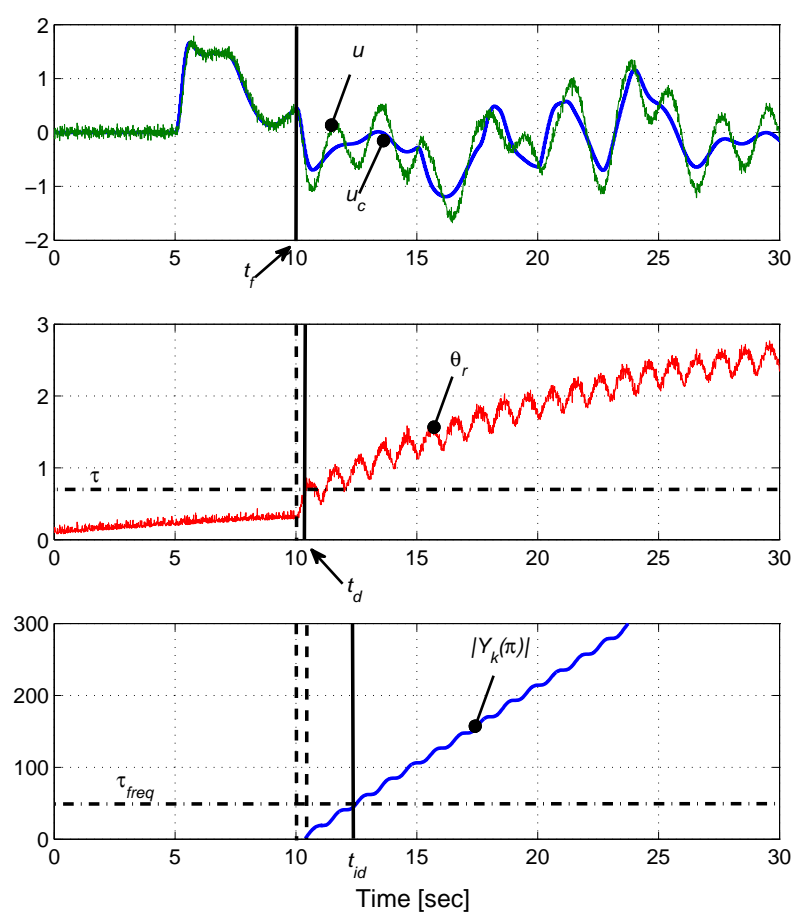

Figure 8: Detection and identification of an OFC

To assess the global performance of the FDD system for the detection of the OFC, the worst-case normalized fault detection and identification time DTP defined in (19) has been determined for several OFC situations in a cruise flight. Recall that the specific choice of the threshold $\tau$ described in subsection 3.3.3 already guarantees the fulfillment of all requirements re- 
garding the lack of false alarms and missed detections, as well as satisfactory detection times. Thus, the performed worst-case analysis only provides supplementary information on the overall robustness of the detection time performance of the FDD system. The worst-case analysis results for three liquid and three solid OFCs for three typical frequencies (small, medium, large) in the range $[0.15,10] \mathrm{Hz}$ are listed in Table 1 . As it can be observed, the DTP tends to increase with the frequency, which indicates that the detection and identification of OFC at higher frequencies appears to be more challenging for the proposed method. Further details can be found in (Varga and Ossmann, 2012).

Table 1: Worst-case analysis results

\begin{tabular}{lcc} 
OFC Type & Frequency & DTP \\
\hline Liquid & small & 0.62 \\
Solid & small & 0.59 \\
Liquid & medium & 0.68 \\
Solid & medium & 0.63 \\
Liquid & large & 0.82 \\
Solid & large & 0.75 \\
\hline
\end{tabular}

\subsection{Monitoring of runaway}

\subsubsection{Parameter tuning}

For the tuning of the FDD system, the values of the parameters $\left(\alpha_{r}, \beta_{r}, \gamma_{r}\right)$ of the residual evaluation block and the threshold $\tau$ of the decision making block have been determined by minimizing the detection time $t_{d}$, under the constraints that no false alarms and no missed detection occur. The optimal setting with $\alpha_{r}=0.6, \beta_{r}=1, \gamma_{r}=0.2$ and the choice $\tau=1$ led to a completely satisfactory fault detection performance.

\subsubsection{Verification and validation results}

The FDD system to detect runaways of the elevator has been tested with the FES. The analysis focus was the determination of the largest DTP over a grid of 316 operating points and parameter combinations, for different runaway rates. The required detection times $t_{\text {detec }}$ can be chosen to correspond to a certain variation of the deflection angle. The analysis in 1530 points determined 0.80 as the worst-case DTP, thus indicating a fast and reliable detection of this type of faults. However, most of DTP values were ranging between $0.1 \div 0.5$, thus providing very satisfactory detection performance. Thus the detection of a runaway fault is very fast as depicted in Fig. 9, which shows the input signal $u_{c}$, the output signal $u$, the evaluation signal $\theta_{r}$ and the corresponding threshold $\tau$, with the fault occurring at $t_{f}$, the fault detection happening at $t_{d}$ and fault identification achieved at $t_{i d}$.

The worst-case search found a value of exactly 1 for the DTP, which results at high absolute values of the runaway speed. The absolute allowed detection time in this case is $0.05 \mathrm{sec}$. For a sampling time $T=0.01 \mathrm{sec}$ this leads to only $n=5$ time steps allowed to accomplish the detection as well as the identification. However, the fault identification can be accomplished for
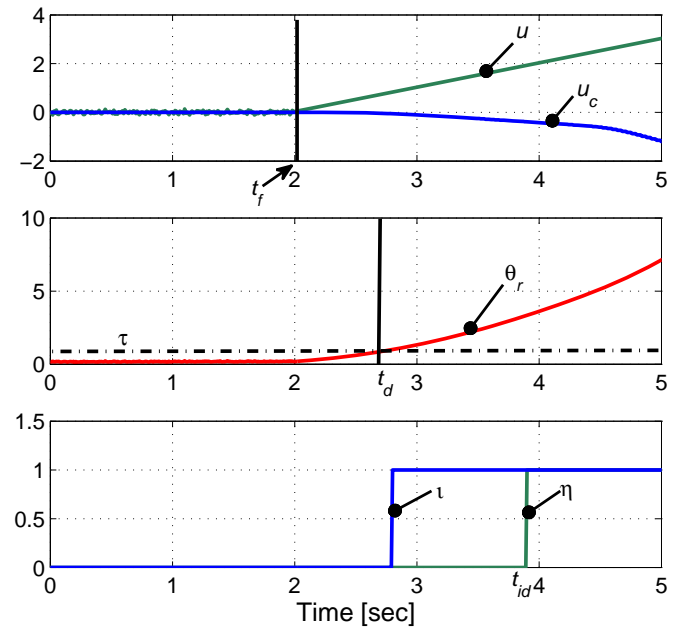

Figure 9: Typical detection of a runaway during cruise

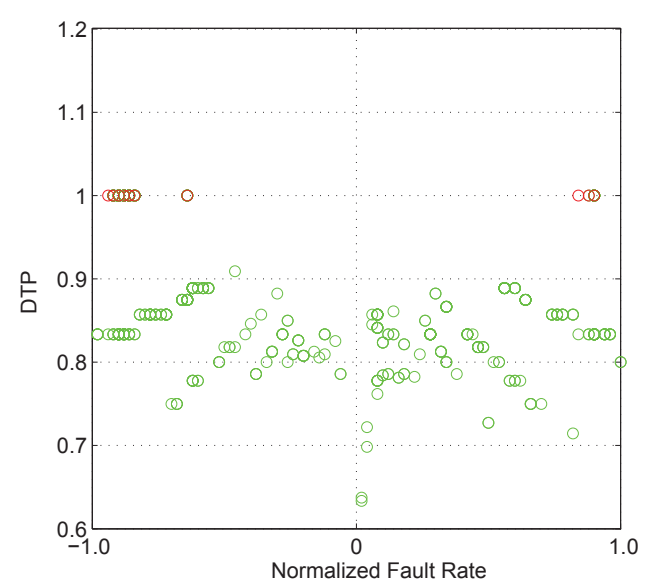

Figure 10: Search point generated by the DE method

high rates in one single time step, as the variation rate of the actuator dominates the measurement noise and the value of $|\bar{u}|$ exceeds $\dot{u}_{\text {max }}$ its threshold after one single time step. The worst case search yields to a DTP including the fault identification time of exactly 1. Fig. 10 depicts search points for different faults rates, which have been generated by the DE method during the worst-case search. Clearly visible is the increase of the DTP with the absolute value of the fault rate, while for low velocities the DTP lies below one.

\subsection{Monitoring of loss of efficiency}

\subsubsection{Parameter tuning}

The free parameters of the fault evaluation and decision block in case of the loss of efficiency have been determined by minimizing the detection time $t_{d}$ again under the constraints that no false alarms and no missed detections occur. The optimal setting for the free parameters was found at $\alpha_{r}=0.05$, $\beta_{r}=1, \gamma_{r}=0.8$. The choice of $\tau=0.1$ satisfies the condition 
$\tau_{f}<\tau \leq \tau_{d}$ and led to a completely satisfactory fault detection performance.

\subsubsection{Verification and validation results}

The robustness of the designed FDD system has been tested for both fault free and faulty situations in the whole flight envelope and full range of aircraft parameter variations. To check for the lack of false alarms, typical maneuvers have been used. The worst-case analysis was conducted to find the largest DTP for a minimum efficiency loss between $30 \%$ and $90 \%$. The requirement was defined to detect the fault before the end of a turn maneuver with very small actuator inputs, thus making the detection difficult. The resulting worst case for the total detection time of the fault was found at $t_{i d}-t_{f}=4.8 \mathrm{sec}$, thus providing satisfactory detection performance. Fig. 11 depicts a typical situation of the loss of efficiency during a turn manoeuver, which shows the input signal $u_{c}$, the output signal $u$, the evaluation signal $\theta_{r}$ and the corresponding threshold $\tau$, with the fault occurring at $t_{f}$, the fault detection happening at $t_{d}$ and fault identification at $t_{i d}$.
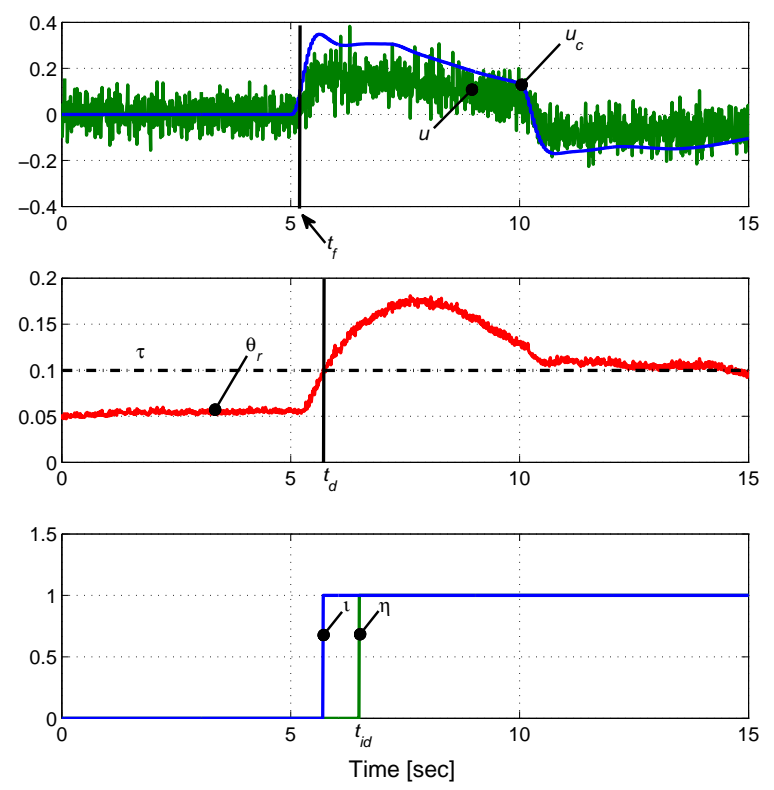

Figure 11: Typical detection and identification of a efficiency loss of $50 \%$ during a smooth turn

\section{Conclusions}

We described and applied a practical model-based methodology for the synthesis of a FDD system for monitoring control surface faults using a component level approach. Several advantages of the proposed methodology are important to be recalled. These are: (1) the employed accurate LPV approximation of the nonlinear actuator model guarantees a robust fault detection performance using a low complexity (first order) LPV fault detection filter; (2) the integrated optimal tuning of the parameters of the fault evaluation and diagnosis blocks in a system-wide closed-loop environment guarantees the overall performance robustness of the FDD system (i.e., lack of false alarms, lack of missed detections, satisfactory detection times); (3) the use of suitable fault identification techniques additionally enhances the robustness of the fault detection and provides useful information for control reconfiguration in a fault tolerant control setting; (4) the low real-time computational burden associated with the designed FDD system is guaranteed by the low complexity (first order) fault detection filter and the triggering of execution of identification algorithms only after the detection of a fault. The proposed FDD system architecture in Fig. 2 can be seen as generic in addressing all kind of additive actuator-control surface faults using a component level approach. A similar architecture for a local monitoring of different categories of parametric (i.e., multiplicative) faults (e.g., actuator rod disconnection or floating, loss of efficiency due to changes in actuator gain) can be devised using model-detection techniques based on a multi-model approach as described in (Varga, 2009a; Ossmann and Varga, 2013).

\section{References}

Alcorta-Garcia, E., Zolghadri, A., Goupil, P., 2011. A nonlinear observer-based strategy for aircraft oscillatory failure detection: A380 case study. IEEE Transactions on Aerospace and Electronic Systems 47, 2792-2806.

Alwi, H., Edwards, C., 2012. Second order sliding mode observers for the ADDSAFE benchmark problem, in: Proc. of SAFEPROCESS'12, Mexico City, Mexico.

Bošković, J., Bergstrom, S.E., Mehra, R., 2005. Adaptive accommodation of failures in second-order flight control actuators with measurable rates, in: Proc. American Control Conference, Portland, OR, USA.

Emami-Naeini, A., Akhter, M.M., Rock, S.M., 1988. Effect of model uncertainty on failure detection: The threshold selector. IEEE Trans. Automat. Control 33, 1106 - 1115

Fielding, C., Varga, A., Bennani, S., Selier, M. (Eds.), 2002. Advanced Techniques for Clearance of Flight Control Laws. volume 283 of Lecture Notes in Control and Information Science, Springer-Verlag, Berlin.

Gheorghe, A., Zolghadri, A., Cieslak, J., Goupil, P., Dayre, R., Berre, H.L., 2013. Model-based approaches for fast and robust fault detection in an aircraft control surface servo loop. Control Systems Magazine 33, 20-30.

Goupil, P., 2010. Oscillatory failure case detection in the A380 electrical flight control system by analytical redundancy. Control Engineering Practice 18, 1110-1119.

Goupil, P., 2011. AIRBUS state of the art and practices on FDI and FTC in flight control system. Control Engineering Practice 19, 524-539.

Goupil, P., Marcos, A., 2012. Industrial benchmarking and evaluation of ADDSAFE FDD designs, in: Proc. of SAFEPROCESS'12, Mexico City, Mexico.

Henry, D., Zolghadri, A., Cieslak, J., Efimov, D., 2012. A LPV approach for early fault detection in aircraft control surfaces servo-loops, in: Proc. of SAFEPROCESS'12, Mexico City, Mexico.

Joos, H.D., 1999. A methodology for multi-objective design assessment and flight control synthesis tuning. Aerospace Science and Technology 3, 161176.

Márton, L., Ossmann, D., 2012. Energetic approach for control surface disconnection fault detection in hydraulic aircraft actuators, in: Proc. of SAFEPROCESS'12, Mexico City, Mexico.

Morelli, E.A., 2000. Real-time parameter estimation in the frequency domain. AIAA Journal of Guidance, Control and Dynamics 23, 812-818.

Narendra, K., Balakrishnan, J., 1997. Adaptive control using multiple models. IEEE Trans. Automat. Control 42, 171-187.

Ossmann, D., Varga, A., 2013. Detection and identification of loss of efficiency faults of flight actuators, in: Proc. of SYSTOL'13, Nice, France.

Stoica, P., Moses, R.L., 1997. Introduction to Spectral Analysis. Prentice Hall. 
Storn, R., Price, K., 1997. Differential evolution - a simple and efficient heuristic for global optimization over continuous spaces. Journal of Global Optimization 11, 341-359.

Vanek, B., Szabó, Z., Edelmayer, A., Bokor, J., 2012. Fault detection of electrical flight control system actuators using parameter dependent estimation, in: Proc. of SAFEPROCESS'12, Mexico City, Mexico.

Varga, A., 2009a. Least order fault and model detection using multi-models, in: Proc. CDC'2009, Shanghai, China.

Varga, A., 2009b. Monitoring actuator failures for a large transport aircraft the nominal case, in: Proc. SAFEPROCESS'2009, Barcelona, Spain.

Varga, A., 2011. On parametric solution of fault detection problems, in: Proc. IFAC 2011 World Congress, Milano, Italy, pp. 6697-6702.

Varga, A., Hansson, A., Puyou, G. (Eds.), 2011a. Optimization Based Clearance of Flight Control Laws. volume 416 of Lecture Notes in Control and Information Science, Springer-Verlag, Berlin.

Varga, A., Hecker, S., Ossmann, D., 2011b. Diagnosis of actuator faults using LPV-gain scheduling techniques, in: Proc. of AIAA Guidance, Navigation, and Control Conference, Portland, Oregon, USA.

Varga, A., Ossmann, D., 2012. LPV-model based identification approach of oscillatory failure cases, in: Proc. of SAFEPROCESS'12, Mexico City, Mexico.

Varga, A., Ossmann, D., Goupil, P., Sabot, G., 2013. Verification and validation of a FDD system for identification of aircraft control surface jamming, in: 19-th IFAC Symposium on Automatic Control in Aerospace, Würzburg, Germany.

Welford, B.P., 1962. Note on a method for calculating corrected sums of squares and products. Technometrics 4, 419-420. 